OPEN ACCESS

Edited by:

Huizi Jin,

Shanghai Jiaotong University, China

Reviewed by:

Wen Zhou,

Guangzhou University of Chinese Medicine, China

Ou Sha,

Shenzhen University, China

*Correspondence:

Li Fang

18321036166@163.com

tThese authors have contributed equally to this work

Specialty section:

This article was submitted to Pharmacology of Anti-Cancer Drugs, a section of the journal Frontiers in Oncology

Received: 28 August 2019 Accepted: 24 February 2020 Published: 02 April 2020

Citation: Zhang L, Huang X, Guo T, Wang $H$,

Fan $H$ and Fang $L$ (2020) Study of Cinobufagin as a Promising Anticancer Agent in Uveal Melanoma Through Intrinsic Apoptosis Pathway. Front. Oncol. 10:325

doi: 10.3389/fonc.2020.00325

\section{Study of Cinobufagin as a Promising Anticancer Agent in Uveal Melanoma Through Intrinsic Apoptosis Pathway}

\author{
Leilei Zhang $^{1,2 \dagger}$, Xiaolin Huang ${ }^{1,2 \dagger}$, Tao Guo ${ }^{1,2}$, Huixue Wang ${ }^{1,2}$, Haiyan Fan ${ }^{1,2}$ and \\ Li Fang $^{1,2 *}$
}

' Department of Ophthalmology, Ninth People's Hospital, Shanghai Jiaotong University School of Medicine, Shanghai, China, ${ }^{2}$ Shanghai Key Laboratory of Orbital Diseases and Ocular Oncology, Shanghai, China

Uveal melanoma $(\mathrm{UM})$ is the most common primary intraocular carcinoma in adults. Cinobufagin, secreted by the Asiatic toad Bufo gargarizans, is a traditional Chinese medicine, widely used in tumor treatment. Here, we explored the potential antitumor function of cinobufagin and investigated its biochemical mechanisms in UM cells. The antitumor potential of cinobufagin was determined via cell viability, cell cycle, and apoptosis assays. Colony formation assays confirmed that cinobufagin exerted potent antitumor activity in a dose-dependent manner. We found that cinobufagin could induce cell apoptosis and upregulate the expression of cleaved caspase-3, cleaved poly(ADP-ribose) polymerase (PARP), and cleaved caspase- 9 in vivo and in vitro. In addition, after treatment with increased concentrations of cinobufagin, the intrinsic mitochondrial apoptosis pathway was also activated, which was demonstrated by increased cell apoptosis with increased expression of Bad and Bax, decreased expression of $\mathrm{Bcl}-2$ and $\mathrm{Bcl}-\mathrm{xl}$, and reduced mitochondrial membrane potential (MMP) in OCM1 cells. Taken together, the results of this preclinical study suggest that cinobufagin can both inhibit cell survival and induce cell apoptosis in a dose-dependent manner in UM cells, which provides new insights into the biochemical mechanism of cinobufagin and its potential as a future chemotherapeutic agent for UM.

Keywords: cinobufagin, anticancer agent, uveal melanoma, apoptosis, MMP

\section{INTRODUCTION}

Uveal melanoma (UM) represents the most frequently occurring primary intraocular malignant carcinoma in adults, with an annual incidence rate of 5.1 cases per million. Lesions are situated either in the choroid (90\%), ciliary body (6\%), or iris (4\%) (1). Currently, the main treatment of $\mathrm{UM}$ is surgery, radiotherapy, and even enucleation. However, the long-term survival rate of uveal melanoma is relatively low due to the risk for liver metastasis. Thus, early detection and treatment of small lesions play a crucial role in achieving not only local disease control and vision preservation but also the possibility of preventing metastasis and improving overall patient survival $(1,2)$.

Traditional Chinese medicine (TCM) provides valuable resources for the treatment of cancers (3). With higher efficiency, weaker side effects, easier access, and better quality of life outcomes, TCMs are widely used in clinics (4). Recently, in the United States and Europe, TCM has been widely used as a complementary and alternative treatment (5). 
Chan-su is a universally known TCM among some Asian countries, which is derived from the dried secretion and parotid glands from the skin of the Asiatic toad Bufo gargarizans (6). Cinobufagin (CBG) is a primary and active component of Chan-su, which has potential anticancer properties (7). Researches have demonstrated that cinobufagin showed antitumor effect in gastric cancer (8), lung cancer cachexia (9), breast cancer (10), osteosarcoma (11), and pancreatic cancer.

Preliminary studies have also demonstrated that CBG could suppress cell growth and induce apoptosis via the Notch pathway in osteosarcoma cells. The expression of Notch1, Hey-1, Hes-1, and Hes-5 was significantly downregulated after treated with cinobufagin in osteosarcoma (OS) cell lines. In addition, the activation of Notch pathway could attenuate CBG-induced apoptosis (11). Another report showed that cinobufagin could induce cell apoptosis through the intrinsic, mitochondrion-dependent apoptosis pathway via the aggregation of reactive oxygen species (ROS) and the loss of mitochondrial membrane potential $(\Delta \Psi \mathrm{m})$ in FOB1.19 and U2OS osteosarcoma cells (12). In U266 human multiple myeloma cells, cinobufagin possibly exerted its antitumor effects via the activation of c-JUN N-terminal kinase (JNK), extracellular signal-regulated kinase (ERK), p38, mitogenactivated protein kinase (MAPK), and caspase-3 mediated through ROS (13). Cinobufagin inhibited the cell growth and triggered apoptosis via the alternation of the expression of Bax and Bcl-2 in human breast cancer MCF-7 cells (10). However, the antitumor activities and biochemical mechanism of cinobufagin in uveal melanoma are still elusive and need to be further elucidated.

\section{MATERIALS AND METHODS}

\section{Chemicals and Reagents}

Cinobufagin was purchased from Anhui Jinchan Biochemical Co., Ltd. (Anhui, China). Cell counting kit-8 (CCK-8) was purchased from Dojindo (Kumamoto, Japan). The primary antibody against $\beta$-actin was purchased from Sigma (St. Louis, MO, United States). Primary antibodies against caspase-3, caspase-9, Bcl-2, and PARP were purchased from Abcam (Cambridge, United Kingdom).

\section{Cell Culture}

The human UM cell line OCM1 was cultivated in Dulbecco's modified Eagle's medium (DMEM) containing $10 \%$ fetal bovine serum (FBS) and 1\% antibiotics (penicillin, 100 $\mathrm{U} / \mathrm{ml}$; streptomycin, $100 \mu \mathrm{g} / \mathrm{ml}$ ). These cells were placed in a humidified environment at $37^{\circ} \mathrm{C}$ with $5 \% \quad \mathrm{CO}_{2}$ and $100 \%$ humidity. The culture medium was replaced every 3 days.

\section{Cell Viability Assay}

OCM1 cells were suspended at a final concentration of $5 \times 10^{3}$ cells/well and cultivated in triplicate in a 96well plate. The viability of cells was measured via the
CCK-8 assay at the indicated time points. Then, CCK$8(10 \mu \mathrm{l})$ was added to each well-containing a $100-\mu \mathrm{l}$ mixture of culture medium. The plate was incubated for $1.5 \mathrm{~h}$ at $37^{\circ} \mathrm{C}$. Viable cells were counted by absorbance measurements at $450 \mathrm{~nm}$ using an automated microplate reader (Tecan Sunrise, Austria). All experiments were performed in triplicate.

\section{Flow Cytometry}

Cells were collected and fixed in $70 \%$ ethanol, treated with $300 \mu \mathrm{g} / \mathrm{ml}$ RNase A (Sigma-Aldrich, St. Louis, MO, United States), and their nuclei were stained with $10 \mu \mathrm{g} / \mathrm{ml}$ propidium iodide (Sigma-Aldrich, St. Louis, MO, United States). The stained nuclei were detected by a FACSCalibur cytometer. The data were analyzed by ModFit software. The apoptosis assay was performed using an Annexin V-FITC apoptosis detection kit (Sigma-Aldrich, St. Louis, MO, United States). Cells were washed twice with phosphate-buffered saline (PBS) buffer and then resuspended in $1 \times$ binding buffer at a concentration of $1 \times 10^{6}$ cells $/ \mathrm{ml}$, and $5 \mu \mathrm{l}$ of Annexin VFITC conjugate and $10 \mu \mathrm{l}$ of propidium iodide (PI) solution were added to each $500-\mu \mathrm{l}$ cell suspension. Cells were then stained with Annexin V-FITC/PI for $10 \mathrm{~min}$ at room temperature. Stained samples were analyzed via FACS Calibur, and the concrete apoptosis rate was determined using FlowJo software (11).

\section{Western Blotting}

Proteins were extracted via protein lysis buffer. Lysates were centrifuged at $12,000 \times \mathrm{g}$ at $4^{\circ} \mathrm{C}$ for $5 \mathrm{~min}$, and the supernatants were collected. The protein concentration was assessed via the bicinchoninic acid protein assay kit. Cell lysates containing 40 $\mu \mathrm{g}$ protein were separated on a $10 \%$ sodium dodecyl sulfatepolyacrylamide gel electrophoresis (SDS-PAGE) gel and then transferred onto polyvinylidene difluoride (PVDF) membranes (Millipore, United States) using a Trans Blot Turbo. Membranes were then blocked in a solution of Tris-buffered saline containing $0.05 \%$ Tween-20 and 5\% skimmed milk for $60 \mathrm{~min}$ at room temperature. Primary antibodies were incubated overnight at $4{ }^{\circ} \mathrm{C}$. The primary antibodies used were anticleaved caspase- 9 $(1: 1,000)$, anticleaved caspase-3 (1:1,000), anti-PARP $(1: 1,000)$, anti-Bcl-2 (1:1,000), anti-Bcl-xl (1:1,000), anti-Bad (1:1,000), anti-Bax (1:1,000), and anti- $\beta$-actin (1:5,000). Horseradish peroxidase-conjugated secondary antibodies were incubated with the membranes for $2 \mathrm{~h}$ at room temperature. Membranes were finally developed via an enhanced chemiluminescence substrate (11).

\section{Mitochondrial Membrane Potential Measurement}

The $\Delta \Psi \mathrm{m}$ was evaluated via a commercial mitochondrial membrane potential assay kit with JC-1 (Invitrogen, Carlsbad, CA, United States). Cells were treated with the suggested concentrations of cinobufagin $(0,0.1,1$, and $3 \mu \mathrm{M})$ in six-wellplates for $24 \mathrm{~h}$ and stained with $\mathrm{JC}-1$ for $15 \mathrm{~min}$ at $37^{\circ} \mathrm{C}$ in the dark. Then, the cells were then harvested, washed twice 
with PBS, and resuspended in $1 \mathrm{ml}$ PBS for subsequent flow cytometry analysis.

\section{Tumor Xenograft Model in Nude Mice}

OCM1 cells $\left(1 \times 10^{7}\right.$ in $200 \mu$ l per injection $)$ were subcutaneously injected into the right flanks of 3-week-old thymic nude mice $(n=20)$. On the ninth day, when the tumor size was $\sim 100 \mathrm{~mm}^{3}$, the nude mice were randomly divided into four groups $(n=5)$, and experimental group 1 began receiving drugs via intraperitoneal injection of CBG at $5 \mathrm{mg} / \mathrm{kg}$ once a day for 10 days. Control group 1 was intraperitoneally injected with $0.5 \mathrm{mg} / \mathrm{kg}$ saline for 10 days. On the fifth day, 10 mice had tumors up to $300 \mathrm{~mm}^{3}$ in size, and experimental group 2 was injected with cinobufagin at $5 \mathrm{mg} / \mathrm{kg}$ once a day for 7 days. Control 2 group was untreated. The mice were housed under a controlled environment in a sterile facility. The tumor volume was measured every 3 days with calipers. Tumor volume was calculated using the formula: $0.5 \times$ length $x$ width $\times$ width. After 30 days, the mice were killed; then, the tumors were removed and analyzed. The Animal Care and Use Committee at Shanghai Jiaotong Medical University approved the animal protocols.

\section{Total RNA Isolation, Reverse Transcription, and Quantitative Polymerase Chain Reaction}

The tissues were immersed in TRIzol reagent (Invitrogen, Carlsbad, CA, United States) and ultrasonicated to extract the total RNA according to the manufacturer's protocol. Complementary DNA (cDNA) was synthesized using the PrimeScriptTM RT reagent Kit (TaKaRa, Tokyo, Japan). The concentration and purity of total RNA were spectrophotometrically determined by the optical density (OD) at $260 \mathrm{~nm}$ and the OD at $280 \mathrm{~nm}$ using a NanoDrop $2000 \mathrm{UV}$ spectrophotometer (Thermo Scientific, United States) (14). The primers for caspase-3, PARP, Bcl-2, Bcl-xl, Bad, and Bax used for reverse transcription quantitative polymerase chain reaction (RT-qPCR) analyses are described in Supplemental Table 1. RT-qPCR was conducted using the Applied Biosystems 7500 Real-Time PCR System (Applied Biosystems, Irvine, CA, United States) and SYBR Premix Ex TaqTM (TaKaRa, Tokyo, Japan). The PCR for each gene was repeated at least three times for each sample, and data were analyzed by comparing the $2^{-\Delta \mathrm{Ct}}$ values. Gene expression levels were normalized to GAPDH (14).

\section{Statistical Analysis}

Statistical analysis was conducted via Prism 5 software (GraphPad Software, Inc., La Jolla, CA, United States). Data were shown as the mean \pm standard error of mean (SEM). The significant difference between the treated and the control was tested by Student's $t$-test. The Kaplan-Meier method was used to estimate the survival curve and the log-rank test to generate statistical significance. $P<0.05$ was considered as statistically significant. Each experiment in this article was repeated at least three times (15).

\section{RESULTS}

\section{Cinobufagin Exerted Potent Cytotoxic and Anticell Growth Activity Against Human UM Cells}

To explore the potential effect of cinobufagin on uveal melanoma cells, a colony formation assay was used to determine the inhibitory effect of cinobufagin. As shown in Figure 1A, cinobufagin had a significant inhibitory effect on OCM1 cells. After treatment with different concentrations $(0,0.03$, 0.1 , and $0.3 \mu \mathrm{M}$ ) of cinobufagin for 1 week, the number of colonies decreased in a dose-dependent manner (Figure 1B). In addition, the CCK-8 cell viability assay showed that the proportions of living cells were approximately $87,77,53$, and $48 \%$ at concentrations of $0.1,0.3,1$, and $3 \mu \mathrm{M}$ cinobufagin, respectively. However, after treatment with 10 and $30 \mu \mathrm{M}$ cinobufagin, the proportion of viable cells was only 37 and $35 \%$, respectively (Figure 1C). These results demonstrated that cinobufagin effectively inhibited OCM1 cell survival. Here, the IC50 value of cinobufagin in OCM1 cells was $0.8023 \mu \mathrm{M}$ after treatment for $48 \mathrm{~h}$. In consideration of the toxicity of cinobufagin, we chose $0.3,1$, and $3 \mu \mathrm{M}$ concentrations for the following experiments.

\section{Cinobufagin Arrested OCM1 Cell Cycle Progression}

The impact of cinobufagin on OCM1 cell cycle progression was measured by flow cytometry. As shown in Figure 2A, after treatment with cinobufagin for $24 \mathrm{~h}$ in OCM1 cells, the percentage of cells in G1 phase significantly increased (Figure 2B). Correspondingly, the percentages of S and G2/M phase cells decreased (Figure 2C). In addition, cinobufagin arrested the cell cycle in the G1 phase in a concentrationdependent manner. It showed that cinobufagin induced cell cycle arrest in OCM1 cells.

\section{Cinobufagin Induced Cell Apoptosis and Alterations of Apoptosis-Related Proteins in OCM1 Cells}

Next, to determine whether cinobufagin influences the apoptosis of OCM1 cells, we utilized the Annexin V-FITC/PI staining method according to a previous study $(16,17)$. Our results showed that cinobufagin could induce OCM1 cell apoptosis with apoptosis rates of approximately 12.6, 56.4, and 63.6\% after treatment with different concentrations of cinobufagin $(0.3,1$, and $3 \mu \mathrm{M})$, while the percentage of apoptotic cells showed no statistically significant difference at a concentration of $0.3 \mu \mathrm{M}$ compared with that in the control group (Figures 3A,B). Furthermore, Western blot analysis showed that cinobufagin significantly upregulated the expression of cleaved caspase3 , cleaved caspase-9, and cleaved PARP in a dose-dependent response (Figures $\mathbf{4 A}, \mathbf{B}, \mathbf{D}, \mathbf{E}$ ). The mitochondrion-dependent apoptosis pathway is controlled by B-cell lymphoma 2 (Bcl2) family proteins (18). Antiapoptotic Bcl-2 and Bcl-xl are important in determining cell death. The low levels of Bcl-2 and $\mathrm{Bcl}-\mathrm{xl}$ could attenuate the inhibitory effect on apoptosis 


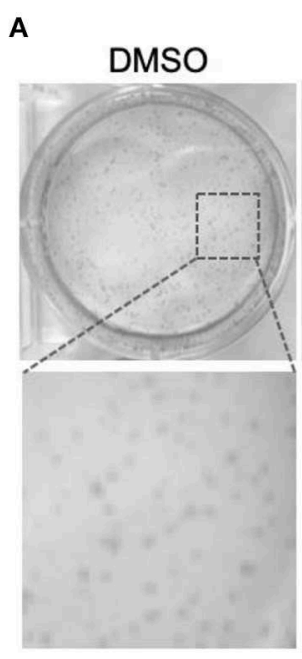

B

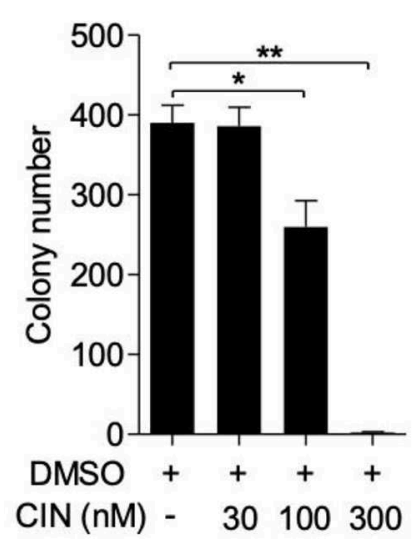

$30 \mathrm{nM}$

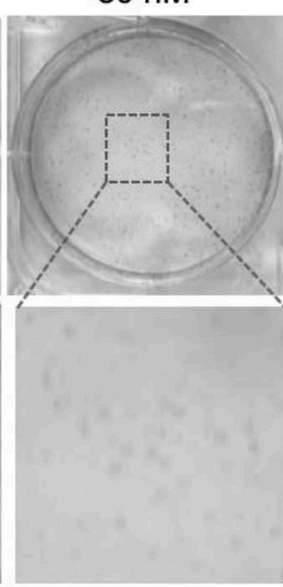

C
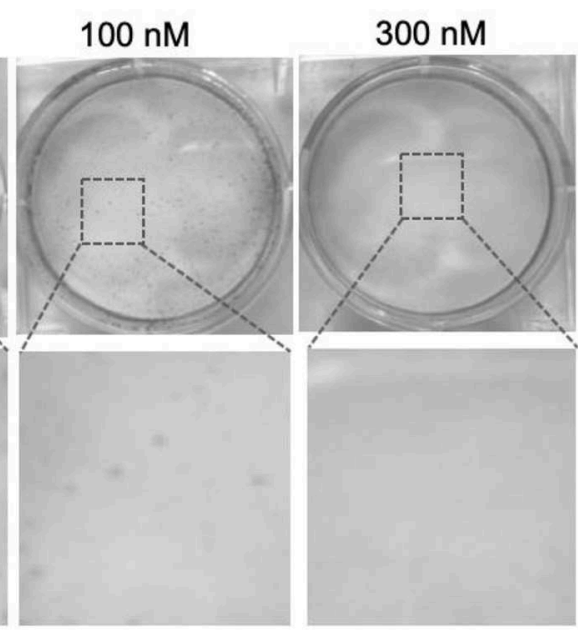

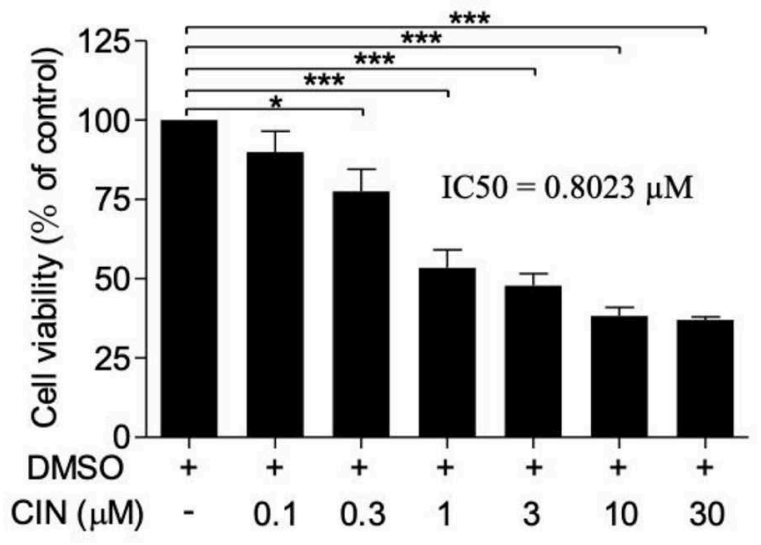

FIGURE 1 | Cinobufagin inhibits OCM1 cell proliferation. (A) Colony formation assay was used to determine the inhibitory effect of cinobufagin (CBG) on OCM1 proliferation. Representative images of colonies from six-well plates by colony formation assay. OCM1 cells were treated with 0, 30,100 , and $300 \mathrm{nM}$ CBG for 1 week. (B) Quantification of the colony number of OCM1 cells. (C) CCK-8 assay was used to detect the toxicity of CBG after treatment for $48 \mathrm{~h}$ on OCM1 cells. The IC50 was calculated to be $0.8023 \mu \mathrm{M}$. The results are expressed as the mean $\pm \mathrm{SEM}$ of three independent experiments $\left[n=3,{ }^{\star} P<0.05,{ }^{* \star} P<0.01\right.$, and ${ }^{\star \star *} P<0.001$ compared with the dimethyl sulfoxide (DMSO) control].

with subsequent induction of the caspase cascade. Here, we observed decreased expression levels of Bcl-2 and Bcl-xl and increased expression levels of proapoptotic proteins Bad and Bax in cinobufagin-treated OCM1 cells (Figures 4A,C,F-H). These data demonstrated that cinobufagin induced cell apoptosis in OCM1 cells via a mitochondrion-dependent pathway.

\section{Cinobufagin Induced Mitochondrial Membrane Potential Depolarization in OCM1 Cells}

The alteration of mitochondrial membrane potential (MMP) is one of the intracellular events that occurs in apoptosis (19). JC1 is an ideal fluorescent probe for detecting MMP. As shown in Figures 5A,B, cinobufagin could decrease the proportion of polymer using the JC-1 indicator. Statistical analysis showed that this effect was dose dependent, with JC-1 aggregates significantly decreasing to 50.4 and $23.4 \%$ in OCM1 cells treated with 1 and $3 \mu \mathrm{M}$ cinobufagin, respectively. The results above demonstrated mitochondrion-mediated cell apoptosis in cinobufagin-treated OCM1 cells.

\section{Cinobufagin Inhibited Xenograft Growth by Inducing Cell Proliferation}

Untreated OCM1 cells were injected into nude mice. On the ninth day, the tumors in groups treated with intraperitoneal injection of cinobufagin and direct injection with cinobufagin grew more slowly than those treated with intraperitoneal injection of saline or untreated (Figure 6A). After intraperitoneal injection of cinobufagin in nude mice for 10 days, tumors in nude mice grew more slowly than those treated with intraperitoneal injection of saline at 24 days. Tumors treated with direct injection for 7 days grew more slowly than those in the control group at 18 days. After 30 days, the mice were executed, and the tumors were removed. The tumors from groups directly injected weighed less than those in the control group (control 2), while differences in the groups treated with 


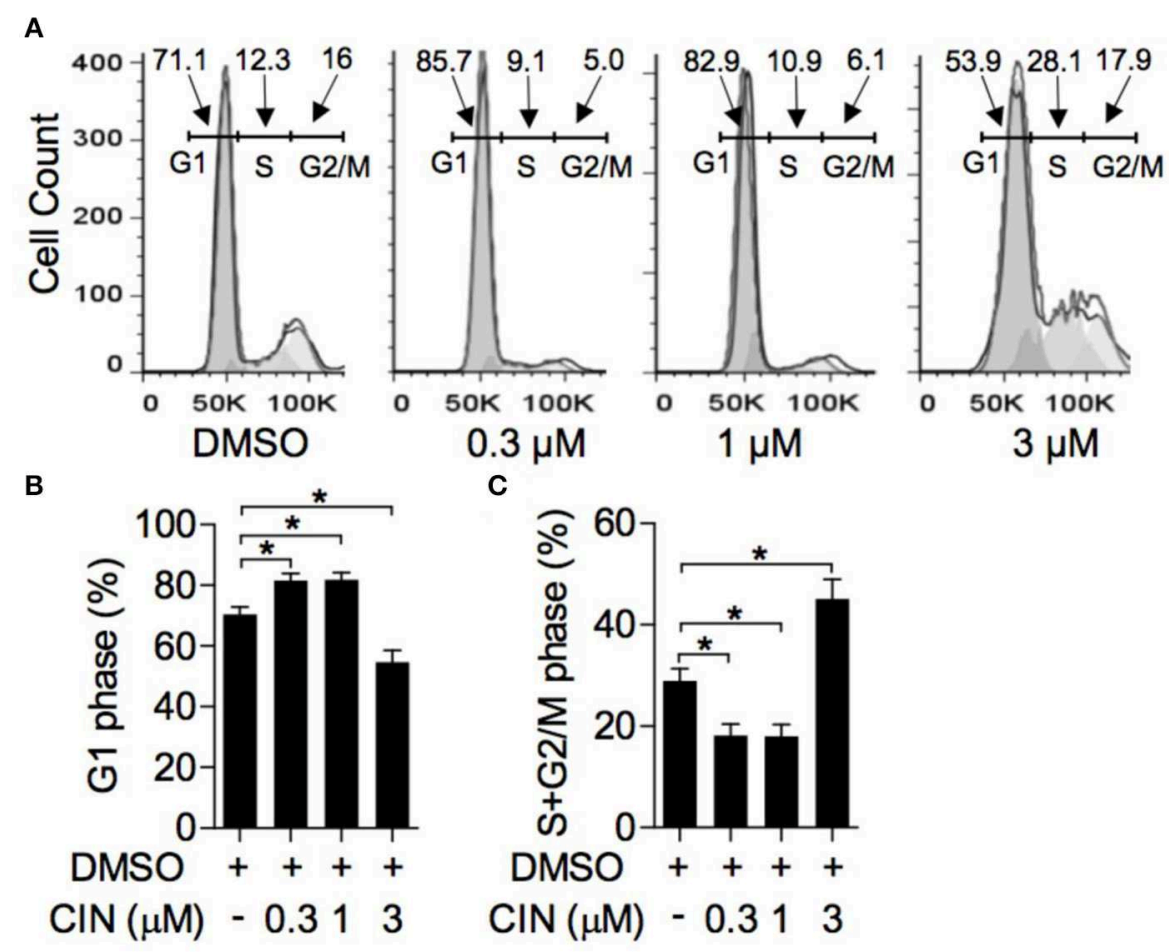

FIGURE 2 | Cinobufagin induces cell cycle arrest of OCM1. (A) OCM1 cells were treated with 0, 0.3, 1, and 3 4 M cinobufagin. After 24 h, OCM1 cells were collected and stained with propidium iodide (PI). Then, the cell cycle distribution was measured with flow cytometry. The percentages of cells in the (B) G1 phase and (C) $\mathbf{S}+$ $\mathrm{G} 2 / \mathrm{M}$ phase were quantified. The results are displayed as the mean $\pm \mathrm{SEM}$ of three independent experiments [ ${ }^{\star} P<0.05$, compared to $\mathrm{OCM} 1 \mathrm{cells}$ incubated with DMSO control].
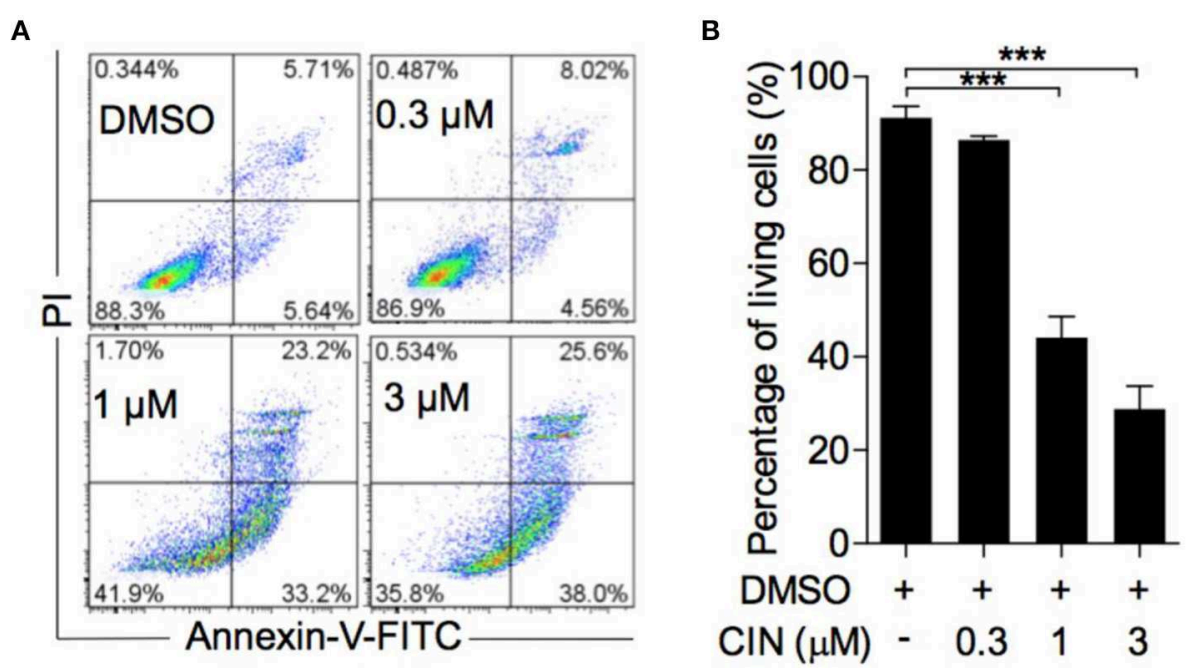

FIGURE 3 | Cinobufagin induces OCM1 cell apoptosis. (A) OCM1 cells were treated with 0, 0.3, 1, and $3 \mu \mathrm{M}$ cinobufagin (CBG) for $24 \mathrm{~h}$. Then, the cells were stained with Annexin V-FITC/PI and measured by flow cytometry. The living cells were located in the lower left quadrant. (B) Quantification of living OCM1 cells. The results are expressed as the mean \pm SEM of three independent experiments [ ${ }^{\star \star \star} P<0.001$ compared with OCM1 cells treated with DMSO].

intraperitoneal injection of cinobufagin were not statistically significant (Figure 6B). RT-qPCR experiments were conducted on the removed tumors. The expression of caspase- 3 and
PARP was increased in tumor tissues (Figures 6C,D). There was decreased $\mathrm{Bcl}-2$ and $\mathrm{Bcl}-\mathrm{xl}$ expression in mouse tumor tissues and increased expression of Bad and Bax (Figures 6E-H). These 
A

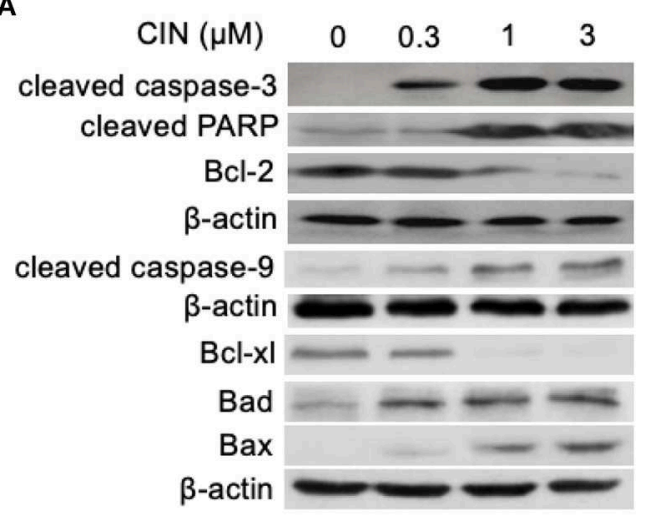

c
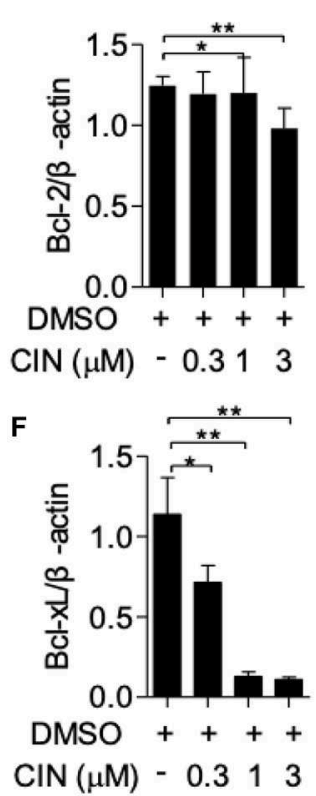

D

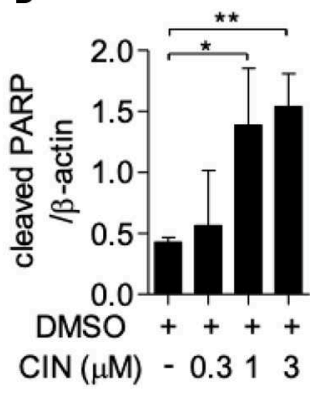

G

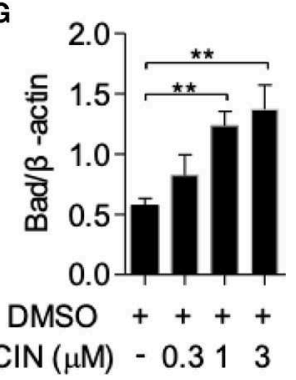

B

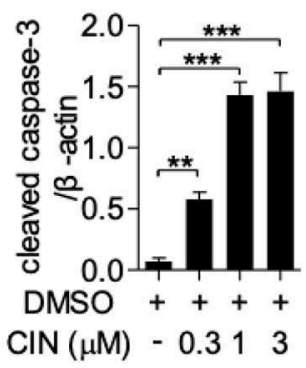

E

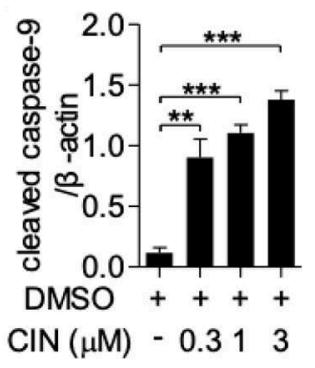

H

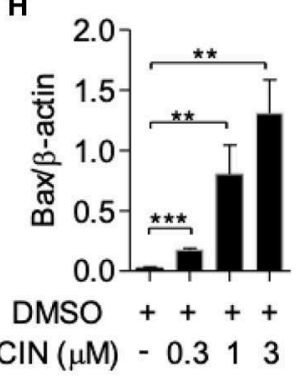

FIGURE 4 | Cinobufagin triggers apoptotic signaling pathways in OCM1 cells. OCM1 cells were treated with 0, 0.3, 1, and 10 $\mu$ M cinobufagin for 24 h. (A) The expression of cleaved caspase-3, cleaved caspase-9, cleaved poly(ADP-ribose) polymerase (PARP), and Bcl-2 was detected by Western blot. $\beta$-Actin was used as a loading control. The expression of (B) cleaved caspase-3, (C) cleaved caspase-9, (D) cleaved PARP, (E) Bcl-2, (F) Bcl-xL, (G) Bad, and (H) Bax was quantified using ImageJ software. The results are shown as the mean \pm SEM of three independent experiments $\left[{ }^{\star} P<0.05\right.$, ${ }^{\star \star} P<0.01$, and ${ }^{\star \star \star} P<0.001$ compared with the DMSO control].

data indicate that proliferation is impaired after cinobufagin treatment in vivo.

\section{DISCUSSION}

At present, neoadjuvant chemotherapy for advanced UM have been adopted largely. However, side effects of chemotherapy and drug resistance cannot be avoided (11). Therefore, it is crucial to identify new chemicals targeting malignant cells. Recently, various novel drugs have been detected. Cinobufagin, secreted by the Asiatic toad B. gargarizans, is a traditional Chinese medicine that has received increasing attention due to its various biological functionalities (20). Whatever, the role of cinobufagin in UM has not been investigated. Therefore, we performed research on whether cinobufagin could inhibit the proliferation and detected the underlying biochemical mechanism in uveal melanoma cells.

Previous researches have demonstrated that cinobufagin exerts antitumor activities by disrupting the cell cycle progression, inducing cell apoptosis, inhibiting angiogenesis, and reversing multidrug resistance $(20,21)$. However, to date, the role of cinobufagin in OCM1 is not well-understood. To determine the functions of cinobufagin on OCM1 cells, we measured the antitumor effect of cinobufagin via a colony formation assay. The results demonstrated that cinobufagin inhibit cell proliferation in a dose-dependent manner in OCM1 cells. In addition, G2/M phrase was arrested, and cell apoptosis was enhanced with higher expression of mitochondrion-related apoptotic proteins after cinobufagin treatment in OCM1 cells. Furthermore, loss of MMP was observed to verify mitochondrion-based cell apoptosis. 

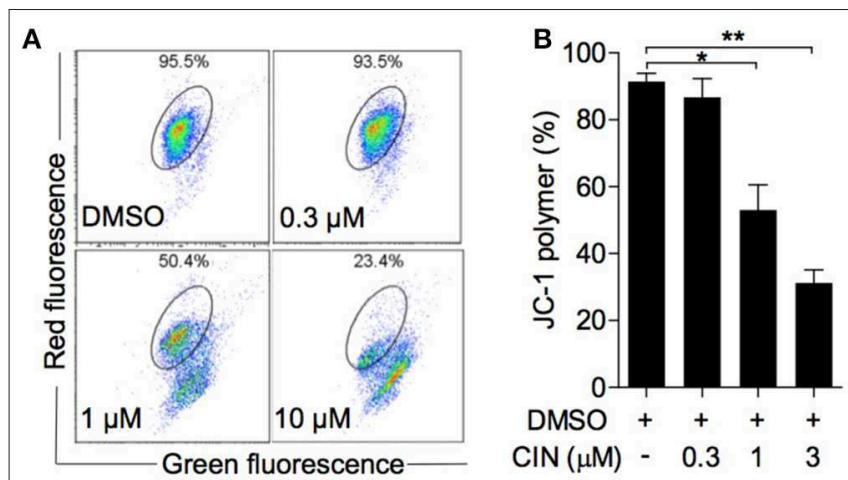

FIGURE 5 | Cinobufagin reduced the mitochondrial membrane potential (MMP) of OCM1 cells. (A) OCM1 cells were treated with cinobufagin at 0, 0.3, 1 , and $10 \mu \mathrm{M}$ for $24 \mathrm{~h}$. Then, OCM1 cells were stained with JC-1, and the change in MMP was measured by flow cytometry. (B) The percentage of aggregated JC-1 was quantified using GraphPad. The results are shown as the mean \pm SEM of three independent experiments $\left[{ }^{\star} P<0.05\right.$ and ${ }^{* \star} P<0.01$ compared with the DMSO group].

Cell apoptosis, also known as programmed cell death, initiates by certain internal or external signals. Apoptosis plays crucial roles in aging, neoplasm, and neurological disorders, such as amyotrophic lateral sclerosis and Parkinson's disease (22), and is characterized by chromatin condensation, subsequent DNA cleavage, and shedding of small fragments from cells (23). Two different pathways are involved in the induction of apoptosis, which occur via the mitochondria (the intrinsic pathway) and/or the activation of death receptors (the extrinsic pathway). Both pathways converge to activate caspases as final executioners of cell death (23). The initialization of apoptosis is a powerful therapeutic strategy in the treatment of various cancers (24). Here, flow cytometry analysis of Annexin V-FITC/PI double-labeled cells revealed that apoptotic rates increased in a concentrationdependent manner after cinobufagin treatment in OCM1 cells. Consistent with our findings, cinobufagin induces apoptosis in a wide range of human tumor cells. Combined, these results confirmed that cinobufagin could induce apoptosis in OCM1 cells.

The mitochondrial apoptotic pathway encompasses the production of ROS, loss of $\Delta \Psi \mathrm{m}$, then triggers the release of cytochrome $\mathrm{c}$ from mitochondria into cytoplasm. The upregulated expression of ROS damages mitochondrial DNA (mtDNA), followed by transcriptional impairment of mtRNAs relevant to the respiratory chain, further enhancing ROS generation (25). Excessive ROS production and oxidative damage to mitochondrial membrane trigger apoptosis and other a series of mitochondrion-associated biological events $(26,27)$. The Bcl-2 family proteins governs the intrinsic mitochondrial apoptotic pathway (18). Antiapoptotic Bcl2 and $\mathrm{Bcl}-\mathrm{xl}$ are important in determining cell fate, and downregulated expression of Bcl-2 causes the loss of $\Delta \Psi \mathrm{m}$ and the subsequent release of cytochrome $\mathrm{c}$ from the mitochondrion into the cytoplasm (28). The proapoptotic proteins Bad and Bax can reside in the cytoplasm, translocate to the mitochondria after receiving death signals, and promote the release of cytochrome $\mathrm{c}$ in mitochondria. Cytochrome c then binds to Apaf-1, which activates caspase-9 to start the caspase cascade where caspase- 3 is cleaved, which triggers the cleavage of numerous key cellular substrates, such as PARP, leading to the fragmentation of DNA and thus inducing cell apoptosis (23). Our results showed the decreased antiapoptotic protein Bcl-2 and Bcl-xl and increased proapoptotic proteins Bad and Bax after treated with cinobufagin in a dose-dependent manner, accompanied with gradual expression of cleaved caspase-9, PARP, and caspase-3 in OCM1 cells.

Animal experiments further demonstrated that cinobufagin could significantly inhibit tumor growth. After 24 days, tumors in nude mice grew more slowly after 10 days of treatment with intraperitoneal injection of cinobufagin than after treatment with intraperitoneal injection of saline. However, compared with control groups, tumors with direct injection for 7 days grew more slowly on the 18th day. Direct injection of cinobufagin into tumors in nude mice was more effective than intraperitoneal injection of cinobufagin when the cinobufagin was at the same concentration. The mice were killed after a treatment duration of 30 days, and the tumors were excised. The directly injected tumors weighed less than the tumors in the control group (control 2), while the tumors treated with intraperitoneal injection of cinobufagin were not significantly different by weight; the concentration of cinobufagin may need to be increased, or there may not have been enough time for cinobufagin to function after intraperitoneal injection. RT-PCR experiments were conducted on the removed tumors. Increased expression of caspase-3, PARP, Bad, and Bax was detected in the tumor tissues. However, compared to control tissues, the expression of $\mathrm{Bcl}-2$ and $\mathrm{Bcl}-\mathrm{xl}$ were decreased in mouse tumor tissues. These data indicate that proliferation is impaired after treated with cinobufagin in vivo.

Furthermore, we assessed the decreased $\Delta \Psi \mathrm{m}$ after cinobufagin treatment. It was supported by the fact that JC-1 aggregate fluorescence waned in cinobufagin-treated OCM1 cells in a dose-dependent manner. However, there were no significant changes in cell apoptosis when treated with $0.3 \mu \mathrm{M}$ cinobufagin. Bcl-2 and cleaved PARP expression were not significantly changed at $0.3 \mu \mathrm{M}$. This means that the mitochondrial/intrinsic pathway was not activated after treatment with $0.3 \mu \mathrm{M}$ cinobufagin. As the caspases were activated at $0.3 \mu \mathrm{M}$, the extrinsic pathway was activated at lower concentrations, which needs further exploration. In general, our data demonstrated that cinobufagin first activates the intrinsic apoptosis pathway to induce OCM1 cell apoptosis.

\section{CONCLUSION}

In conclusion, this study revealed the potential antitumor role of cinobufagin in treating UM and encourages further anticancer research on cinobufagin, which could lead to further studies of cinobufagin for clinical usage in UM. 
A

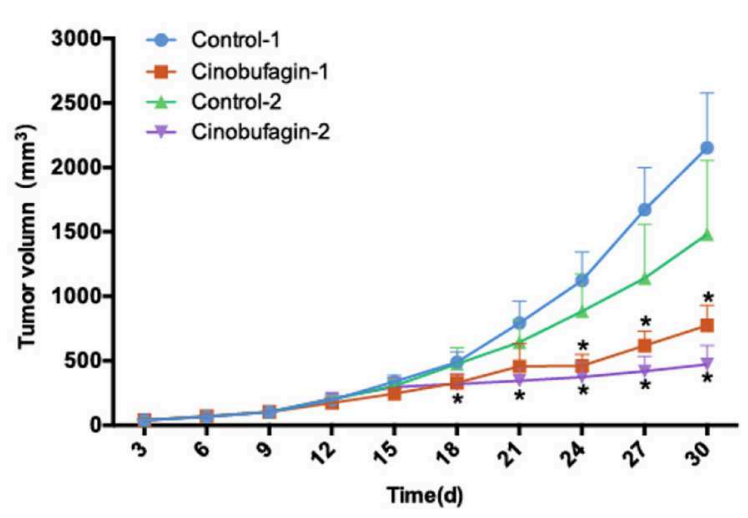

B

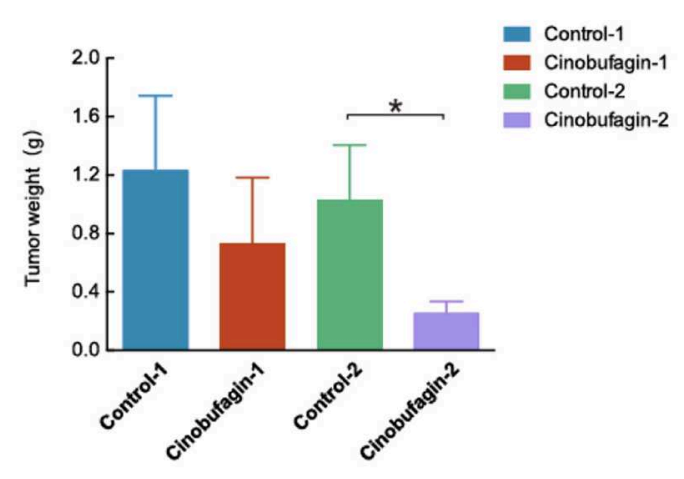

C

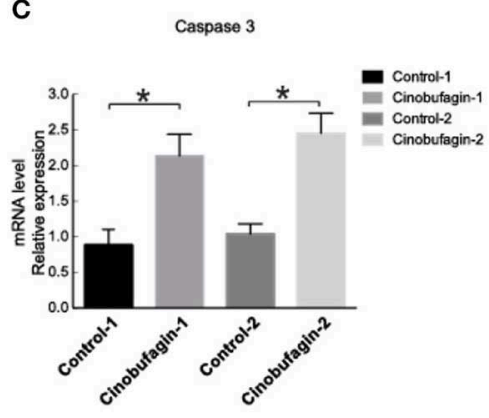

F

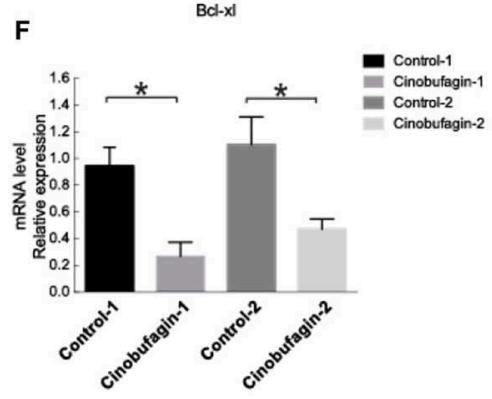

D

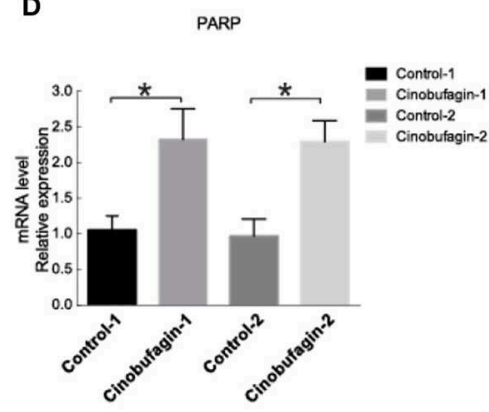

$\operatorname{Bax}$

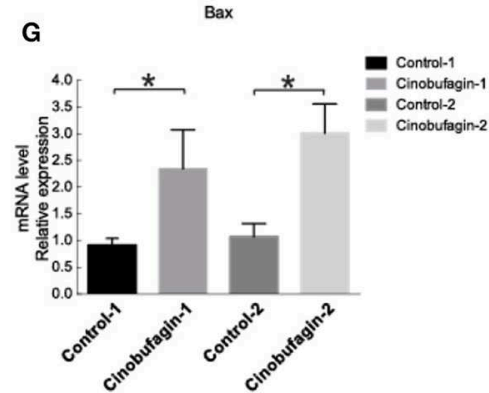

E

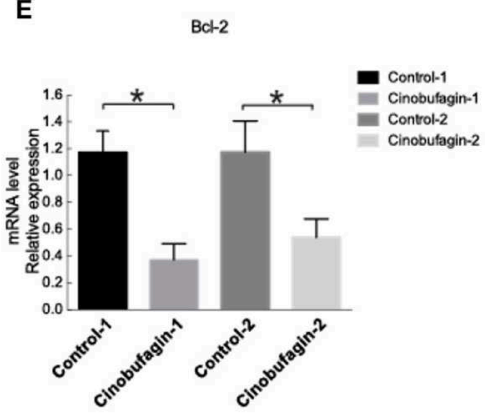

Bad

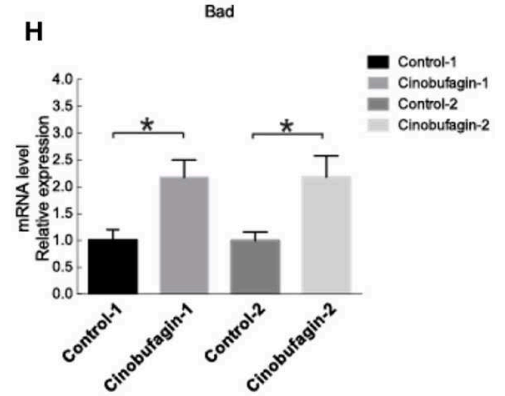

FIGURE 6 | Cinobufagin modulates tumor growth and apoptosis. (A) Nu/Nu nude mice were injected with OCM1 cells. After the tumor size reached 100 mm³ , the experimental group was intraperitoneally injected with cinobufagin at $5 \mathrm{mg} / \mathrm{kg}$ once a day for 10 days (cinobufagin 1). The control group (control 1 ) was intraperitoneally injected with $0.5 \mathrm{mg} / \mathrm{kg}$ saline for 10 days. After the remaining 10 mice had tumors up to $300 \mathrm{~mm}^{3}$, the tumors were injected with cinobufagin at $5 \mathrm{mg} / \mathrm{kg}$ once a day for 7 days (cinobufagin 2). The control 2 group was untreated (control 2). Tumor volume was measured every 3 days. (B) After 30 days, the mice were killed, and the tumors were removed and analyzed. The tumors treated with cinobufagin weighed less. The expression of (C) caspase-3, (D) PARP, (E) Bcl-2, (F) Bcl-xL, (G) Bad, and $(\mathbf{H})$ Bax was quantified by RT-qPCR in the tumors of the mice. The results are shown as the mean \pm SEM of five independent experiments $\left({ }^{*} P<0.05\right)$.

\section{DATA AVAILABILITY STATEMENT}

All datasets generated for this study are included in the article/Supplementary Material.

\section{AUTHOR CONTRIBUTIONS}

LF and LZ contributed conception, design of the study, and wrote sections of the manuscript. LF, TG, HW, and LZ organized the database. LF, TG, HF, and LZ performed the statistical analysis. LZ wrote the first draft of the manuscript. All authors contributed to manuscript revision, read, and approved the submitted version.

\section{FUNDING}

This work was supported by grants from National Natural Science Foundation of China (81972524, 81602366, and 81402258) and the Science and Technology Commission of Shanghai (17DZ2260100), and fund of the Ninth People's Hospital Affiliated to Medical College of Shanghai Jiaotong University (SYZ2015-010).

\section{SUPPLEMENTARY MATERIAL}

The Supplementary Material for this article can be found online at: https://www.frontiersin.org/articles/10.3389/fonc. 2020.00325/full\#supplementary-material 


\section{REFERENCES}

1. Kaliki S, Shields LC. Uveal melanoma: relatively rare but deadly cancer. Eye. (2017) 31:241-57. doi: 10.1038/eye.2016.275

2. Dogrusoz M, Jager MJ, Damato B. Uveal melanoma treatment and prognostication. Asia Pac J Ophthalmol. (2017) 6:186-96. doi: 10.22608/APO.201734

3. Xu H, Zhao X, Liu X, Xu P, Zhang K, Lin X. Antitumor effects of traditional Chinese medicine targeting the cellular apoptotic pathway. Drug Des Devel Ther. (2015) 9:2735-44. doi: 10.2147/DDDT.S80902

4. Xia J, Chen J, Zhang Z, Song P, Tang W, Kokudo N. A map describing the association between effective components of traditional Chinese medicine and signaling pathways in cancer cells in vitro and in vivo. Drug Discov Ther. (2014) 8:139-53.

5. Gai RY, Xu HL, Qu XJ, Wang FS, Lou HX, Han JX, et al. Dynamic of modernizing traditional Chinese medicine and the standards system for its development. Drug Discov Ther. (2008) 2:2-4. doi: 10.1126/science.281.53 85.1967

6. Wang D, Ru W, Xu Y, Zhang J, He X, Fan G, et al. Chemical constituents and bioactivities of Colla corii asini. Drug Discov Ther. (2014) 8:201-7. doi: 10.5582/ddt.2014.01038

7. Lu XS, Qiao YB, Li Y, Yang B, Chen MB, Xing GC. Preclinical study of cinobufagin as a promising anti-colorectal cancer agent. Oncotarget. (2017) 8:988-98. doi: 10.18632/oncotarget.13519

8. Xiong X, Lu B, Tian Q, Zhang H, Wu M, Guo H, et al. Inhibition of autophagy enhances cinobufagininduced apoptosis in gastric cancer. Oncol Rep. (2019) 41:492-500. doi: 10.3892/or.2018.6837

9. Xie M, Chen X, Qin S, Bao Y, Bu K, Lu Y. Clinical study on thalidomide combined with cinobufagin to treat lung cancer cachexia. J Cancer Res Ther. (2018) 14:226-32. doi: 10.4103/0973-1482.188436

10. Zhu L, Chen Y, Wei C, Yang X, Cheng J, Yang Z, et al. Anti-proliferative and pro-apoptotic effects of cinobufagin on human breast cancer MCF7 cells and its molecular mechanism. Nat Prod Res. (2018) 32:493-7. doi: 10.1080/14786419.2017.1315575

11. Cao Y, Yu L, Dai G, Zhang S, Zhang Z, Gao T, et al. Cinobufagin induces apoptosis of osteosarcoma cells through inactivation of Notch signaling. Eur $J$ Pharmacol. (2017) 794:77-84. doi: 10.1016/j.ejphar.2016.11.016

12. Dai G, Zheng D, Guo W, Yang J, Cheng YA. Cinobufagin induces apoptosis in osteosarcoma cells via the mitochondria-mediated apoptotic pathway. Cell Physiol Biochem. (2018) 46:1134-47. doi: 10.1159/000488842

13. Baek SH, Kim C, Lee JH, Nam D, Lee J, Lee SG, et al. Cinobufagin exerts anti-proliferative and pro-apoptotic effects through the modulation ROSmediated MAPKs signaling pathway. Immunopharmacol Immunotoxicol. (2015) 37:265-73. doi: 10.3109/08923973.2015.1027916

14. Zhang L, Huang X, Zhu X, Ge S, Gilson E, Jia R, et al. Differential senescence capacities in meibomian gland carcinoma and basal cell carcinoma. Int $J$ Cancer. (2016) 138:1442-52. doi: 10.1002/ijc.29882

15. Zhang L, Jia R, Zhao J, Fan J, Zhou Y, Han B, et al. Novel mutations in the RB1 gene from Chinese families with a history of retinoblastoma. Tumor Biol. (2014) 36:2409-20. doi: 10.1007/s13277-014-2851-7

16. Jia R, Jiao Z, Xu X, Wang J, Zhou Y, Song X, et al. Functional significance of B7-H1 expressed by human uveal melanoma cells. Mol Med Rep. (2011) 4:163-7. doi: $10.3892 / \mathrm{mmr} .2010 .397$
17. Pan Z, Zhang X, Yu P, Chen X, Lu P, Li M, et al. Cinobufagin induces cell cycle arrest at the G2/M phase and promotes apoptosis in malignant melanoma cells. Front Oncol. (2019) 9:853. doi: 10.3389/fonc.2019.00853

18. Redza-Dutordoir M, Averill-Bates AD. Activation of apoptosis signalling pathways by reactive oxygen species. Biochim Biophys Acta. (2016) 1863:297792. doi: 10.1016/j.bbamcr.2016.09.012

19. Xia J, Inagaki Y, Gao J, Qi F, Song P, Han G, et al. Combination of cinobufacini and doxorubicin increases apoptosis of hepatocellular carcinoma cells through the fas- and mitochondria-mediated pathways. Am J Chin Med. (2017) 45:1537-56. doi: 10.1142/S0192415X17500835

20. Emam H, Zhao QL, Furusawa Y, Refaat A, Ahmed K, Kadowaki M, et al. Apoptotic cell death by the novel natural compound, cinobufotalin. Chem Biol Interact. (2012) 199:154-60. doi: 10.1016/j.cbi.2012.07.005

21. Afroze SH, Sloan J, Osuji GC, Drever N, Pilkinton K, Zawieja DC, et al. Cinobufotalin impedes Sw.71 cytotrophoblast cell line function via cell cycle arrest and apoptotic signaling. Mol Cell Biochem. (2016) 422:189-96. doi: 10.1007/s11010-016-2820-0

22. Fuchs Y, Steller H. Programmed cell death in animal development and disease. Cell. (2011) 147:742-58. doi: 10.1016/j.cell.2011.11.045

23. Fuchs Y, Steller H. Live to die another way: modes of programmed cell death and the signals emanating from dying cells. Nat Rev Mol Cell Biol. (2015) 16:329-44. doi: 10.1038/nrm3999

24. Yaacoub K, Pedeux R, Tarte K, Guillaudeux T. Role of the tumor microenvironment in regulating apoptosis and cancer progression. Cancer Lett. (2016) 378:150-9. doi: 10.1016/j.canlet.2016.05.012

25. Dickinson BC, Chang JC. Chemistry and biology of reactive oxygen species in signaling or stress responses. Nat Chem Biol. (2011) 7:504-11. doi: 10.1038/nchembio.607

26. Mates JM, Segura JA, Alonso FJ, Marquez J. Oxidative stress in apoptosis and cancer: an update. Arch Toxicol. (2012) 86:1649-65. doi: 10.1007/s00204-012-0906-3

27. Fogg VC, Lanning NJ, Mackeigan PJ. Mitochondria in cancer: at the crossroads of life and death. Chin J Cancer. (2011) 30:526-39. doi: $10.5732 / \mathrm{cjc} .011 .10018$

28. Jiang X, Jiang H, Shen Z, Wang X. Activation of mitochondrial protease OMA1 by Bax and Bak promotes cytochrome c release during apoptosis. Proc Natl Acad Sci USA. (2014) 111:14782-7. doi: 10.1073/pnas.1417 253111

Conflict of Interest: The authors declare that the research was conducted in the absence of any commercial or financial relationships that could be construed as a potential conflict of interest.

The handling Editor declared a shared affiliation, though no other collaboration, with all the authors.

Copyright (c) 2020 Zhang, Huang, Guo, Wang, Fan and Fang. This is an open-access article distributed under the terms of the Creative Commons Attribution License (CC $B Y)$. The use, distribution or reproduction in other forums is permitted, provided the original author(s) and the copyright owner(s) are credited and that the original publication in this journal is cited, in accordance with accepted academic practice. No use, distribution or reproduction is permitted which does not comply with these terms. 